\title{
Single-Port Laparoscopic Liver Resection: Largest Turkish Experience
}

\author{
Ilhan Karabicak ${ }^{1} \cdot$ Kagan Karabulut $^{1} \cdot$ Savas Yuruker $^{1}$ • \\ Tugrul Kesicioglu ${ }^{1} \cdot$ Necati Ozen $^{1}$
}

Received: 10 September 2015 / Accepted: 28 December 2015 /Published online: 11 January 2016

(C) Association of Surgeons of India 2016

\begin{abstract}
Single-port laparoscopic surgery has the advantage of a hidden scar and reduced abdominal wall trauma. Although single-port laparoscopic surgery is widely performed for other organs, its application is very limited for liver resection. Here, we report our experience with nine patients who underwent single-port laparoscopic liver resection. Nine patients underwent single-port laparoscopic liver resection for the indications of hydatid cyst, hepatocellular carcinoma, and colorectal cancer liver metastasis. Nine patients were successfully treated with single-port laparoscopic surgery. The operative time was between 60 and $240 \mathrm{~min}$. The only operative complication was bleeding up to $650 \mathrm{~mL}$ in a patient with cirrhosis. No postoperative complications occurred. All patients were discharged earlier than usual. Single-port laparoscopic liver surgery is a challenging surgery. Surgeon with the experience of laparoscopic liver surgery should perform the single-port laparoscopic liver surgery. It is technically feasible with a good outcome in well-selected patients. Initial cases must be benign lesions to avoid jeopardizing oncological safety.
\end{abstract}

Keywords Single port $\cdot$ Laparoscopy $\cdot$ Liver $\cdot$ Cancer . Minimal invasive

Ilhan Karabicak

ikarabicak@yahoo.com

1 Medical Faculty, Department of General Surgery, Ondokuz Mayis University, Atakum, Samsun 55303, Turkey

\section{Introduction}

Laparoscopic liver resection has been the standard treatment for peripherally located benign or malignant lesions in well-selected patients [1-3]. This procedure has the advantage of reducing postoperative general complications such as ascites and wound infection without worsening the oncological outcome. Laparoscopy is preferred over conventional surgery in well-selected patients with cirrhosis with peripherally located tumors to reduce the postoperative deterioration [3-5].

Single-port laparoscopic surgery (SPLS) is a new trend in laparoscopic surgery that has the same outcome as laparoscopy but has additional advantages such as a hidden, scarless incision, and less abdominal trauma [6,7]. Single-port laparoscopic surgery has been successfully performed for gallbladder, bariatric surgery, and colon resections [7-9]. However, the development of this procedure for liver resection has been very slow, similar to the slow progress of laparoscopic liver surgery.

SPL liver surgery has a steep learning curve. Surgeons who have experience with laparoscopic and open liver surgery should perform SPL liver resection after gaining enough experience with other types of resection such as SPL cholecystectomy [10-12].

The indications, contraindications, and technical difficulties such as instrument crowding, absence of triangulation, parallel field of view, and two- to three-instrument restriction depending on the port choice have been discussed in detail in previous reports [10-15].

We reported the first single-port laparoscopic pericystectomy for hydatid cyst [16]. That was our first SPL liver resection, and then we performed eight liver resections.

In this report, we share our experience of nine patients of the SPL liver resection. 


\section{Methods}

From May 2011 to April 2015, nine patients underwent SPL liver resection. The liver lesions were as follows: three hydatid cysts, four colorectal cancer liver metastases, and two hepatocellular carcinomas (HCCs). The patients' preoperative demographics are shown in Table 1.

\section{Results}

We performed two SPL pericystectomies and one left lateral sectionectomy for the hydatid cysts, two metastasectomies and one left lateral sectionectomy for the colorectal cancer liver metastases, one segment 3 resection, and one wedge resection for the HCCs.

One patient with cirrhosis had intraoperative bleeding and required 2 units of erithrocyte transfusion. There were no other perioperative or postoperative complications. The type of surgery and the perioperative and postoperative findings are shown in Table 2.

\section{SPLS for Hydatid Cyst (Cases I, II, and V)}

Cases I, II, and V had a 5, 10, and $4 \mathrm{~cm}$ hydatid cyst, respectively. The cyst occupied all of the left lateral section in patient II. We performed SPL pericystectomy on the first and fifth cases and SPL left lateral sectionectomy (LLS) on the second case.

Meticulous dissection is required to avoid perforating the hydatid cyst, which may cause anaphylaxis. We placed a hydrogen peroxide immersed gauze under the cyst to prevent spillage into the peritoneum and used the gauze for bleeding control and traction if necessary.

Table 1 General data for the nine patients

\begin{tabular}{lllllll}
\hline No. & Age & Sex & Diagnosis & LT & Size $(\mathrm{cm})$ & CP \\
\hline I & 71 & F & Hydatid cyst & Segment 3 & 6 & A \\
II & 50 & F & Hydatid cyst & Segments 2 and 3 & 10 & A \\
III & 58 & M & HCC & Segment 2 & 3 & B \\
IV & 46 & F & Metastasis & Segment 2/3 & 3 & A \\
V & 26 & F & Hydatid cyst & Segment 3 & 4 & A \\
VI & 55 & M & Metastasis & Segment 3 & 2 & A \\
VII & 70 & M & HCC & Segment 3 & 3 & A \\
VIII & 71 & F & Metastasis & Segment 3 & 3 & A \\
IX & 70 & M & Metastasis & Segment 3 & 3 & A \\
\hline
\end{tabular}

$L T$ location of the tumor, $C P$ Child-Pugh classification
Table 2 Type of surgery, perioperative and postoperative findings

\begin{tabular}{lllll}
\hline No: & Type of surgery & Duration $(\mathrm{min})$ & Bleeding $(\mathrm{mL})$ & Complication \\
\hline 1 & Pericystectomy & 80 & $<100$ & No \\
2 & LLS & 220 & 200 & No \\
3 & Wedge resection & 90 & $<100$ & No \\
4 & LLS & 120 & 200 & No \\
5 & Pericystectomy & 60 & $<50$ & No \\
6 & Metastasectomy & 110 & $<100$ & No \\
7 & Wedge resection & 250 & 650 & No \\
8 & Metastasectomy & 90 & $<100$ & No \\
9 & Metastasectomy & 60 & $<100$ & No \\
\hline
\end{tabular}

$L L S$ left lateral sectionectomy

After the SPL pericystectomy was performed, the cyst was placed in a retrieval bag. Then, the SILS port was removed and the retrieval bag was pulled up to the skin incision. The cyst content was aspirated to shrink the cyst while it was in the bag, which enabled the bag to be withdrawn without enlarging the single port incision.

A 2.5-cm SILS port (Covidien, Boulder, CO, USA) is usually adequate for pericystectomy, but a larger port may be necessary if liver tissue will be removed with the cyst.

\section{SPLS for HCC (Cases III and VII)}

Patients IV and VIII had a $2 \mathrm{~cm} \mathrm{HCC} \mathrm{in} \mathrm{segment} 2$ and a $3 \mathrm{~cm}$ HCC in segment 3, respectively (Fig. 1a). We performed a wedge resection on patient IV and partial segment 3 resection on patient VIII. If preoperative studies show large umbilical veins, the single-port incision should not be placed in the umbilicus.

Parenchymal resection is the Achilles' heel of SPL liver resection in a cirrhotic liver. The limited liver resection such as wedge resection can easily be achieved with laparoscopic surgery. The disadvantages of SPLS such as instrument crowding and the absence of triangulation make SPL liver resection crucial in patients with cirrhosis. Bigger single ports and articulating laparoscopic instruments must be used to overcome this disadvantage. These ports have four entries; three instruments can be used at the same time. Articulating laparoscopic ultrasonic surgical shears (Ethicon Endo-Surgery, Cincinnati, OH, USA) will be helpful to avoid resecting excessive liver tissue (Fig. 1b). 

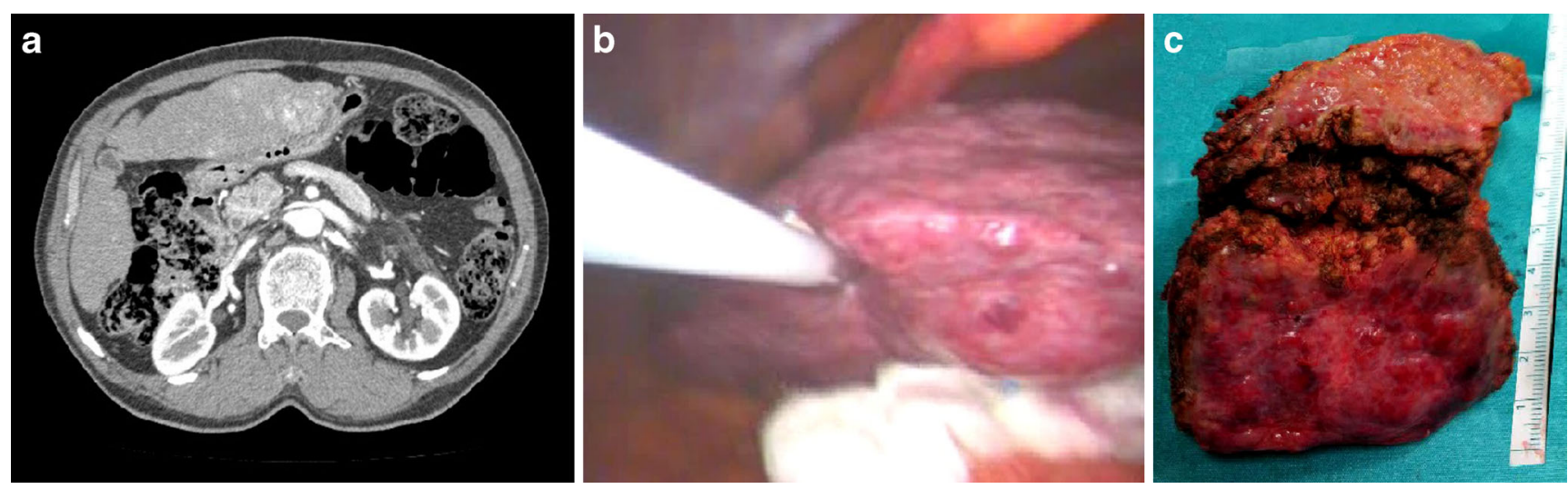

Fig. 1 a A $3 \mathrm{~cm} \mathrm{HCC}$ in segment 3. b Excessive liver tissue is removed since articulation could not be provided with conventional laparoscopic ultrasonic surgical shears

\section{SPLS for Metastatic Liver Tumors (Cases IV, VI, VIII, and IX)}

The fourth, sixth, eighth, and ninth patients were colorectal cancer liver metastases. The fourth case was a metastatic liver tumor diagnosed 2 months after conventional left colectomy. The tumor was between segments 2 and 3 and had invaded the portal branch (Fig. 2a).

If left lateral sectionectomy is planned, the single-port incision must be $5 \mathrm{~cm}$. This incision will be needed to withdraw the left lateral section especially in malignant lesions. Single ports with four entries must be used since all entries will be necessary at the same time for dissection, camera, and traction. The laparoscopic liver retractor is very helpful for traction during left hepatic vein transaction (Fig. 2b). We do not recommend to smash the liver even if the lesion is benign (Fig. 2c).

If there is a previous supraumbilical incision, a $5-\mathrm{cm}$ single-port incision is helpful for sharp and blunt dissection. It is better to make a sharp and blunt dissection before the single port is placed since adhesions may not allow appropriate placement of the port or enough room to manipulate the instruments intracorporeally. Articulating laparoscopic instruments and vascular staplers may be necessary to control inflow and outflow vessels. Articulating vascular staplers are particularly useful in dividing the major trunk of hepatic veins deep in the transection plane.

The sixth, eighth, and ninth patients had peripherally located 2, 2, and $3 \mathrm{~cm}$ colorectal cancer liver metastases in segment 3 , respectively. We performed SPL metastasectomy on all patients by using the SILS port (Fig. 3a-c).

\section{Discussion}

The aim of modern surgery is to go scarless with the same oncological outcome as conventional surgery. Single-port laparoscopic surgery is gaining popularity in which the incision scar is usually hidden in the umbilicus.

SPL liver surgery has a steep learning curve. SPL liver resection must be performed by surgeons experienced in laparoscopic and conventional hepatobiliary surgery [1-3]. The
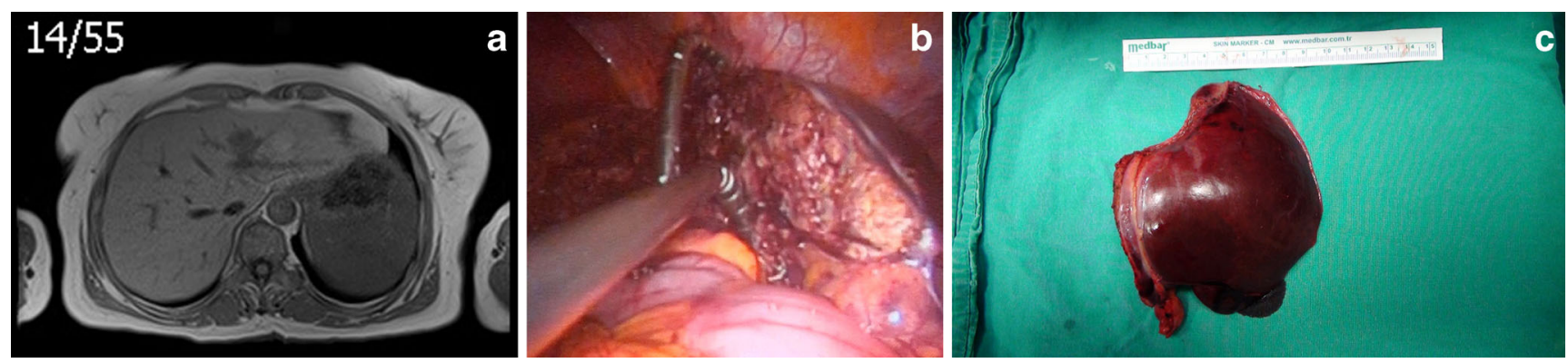

Fig. 2 a A metastaic liver tumor between segments 2 and 3 and invading the portal branch. b The laparoscopic liver retractor is helpful for traction during left hepatic vein transection. c A left lateral section removed by SPLS 

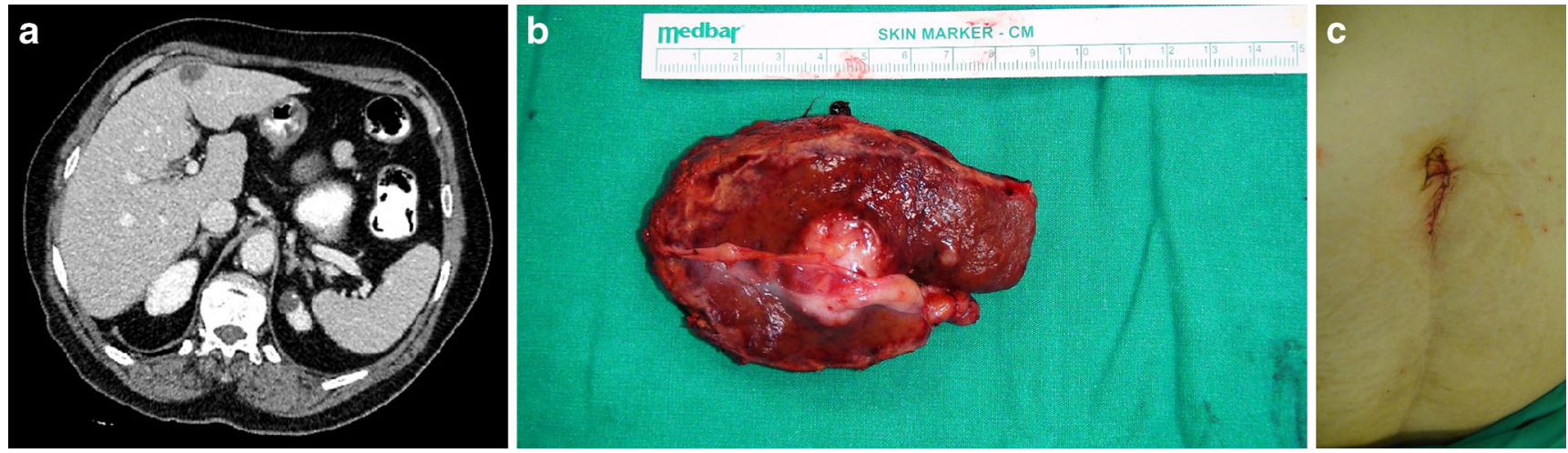

Fig. 3 a A $3 \mathrm{~cm}$ colorectal cancer liver metastases in segment 3. b SPL metastasectomy for colorectal cancer liver metastasis. c A $3 \mathrm{~cm}$ SILS port incision which is created on previous incision scar

initial cases must be selected very carefully to avoid failure during the learning curve, which will cause disappointment. We recommend starting with peripherally located benign liver lesions to avoid jeopardizing the oncological safety during the learning curve. Therefore, our first two cases were liver hydatid cysts.

The benefits of SPL liver resection on a patient with cirrhosis have been demonstrated [4, 10]. Single-port laparoscopic liver resection on a patient with cirrhosis has the main advantage of reduced postoperative deterioration. Patients with cirrhosis must be chosen very carefully to perform SPL liver resection since this technique can cause severe bleeding because of the technical difficulty $[4,12]$. The bleeding can be from the major vessels or the cut surface. Articulating laparoscopic ultrasonic surgical shears and laparoscopic Cavitron Ultrasonic Surgical Aspirator (CUSA, Tyco Healthcare, Mansfield, MA, USA) can reduce deep parenchymal bleeding in patients with cirrhosis.

If the surgery takes a long time or excessive bleeding occurs, the surgeon should not be stubborn to finish the case with a single port, which can harm the patient. Multiport laparoscopic surgery is an alternative to SPLS if excessive bleeding occurs or one cannot overcome the technical difficulty.

Patients with peripherally located small hydatid cysts are good candidates to start performing SPLS. SPL pericystectomy is the accepted gold standard for peripherally located small hydatid cysts [16]. We discharged the third patient with hydatid cyst on whom we had performed SPL pericystectomy on postoperative day 1 . In such cases, SPLS is similar to ambulatory surgery.

\section{Conclusion}

Single-port laparoscopic liver resection is gaining popularity at experienced centers $[17,18]$. The small case series demonstrated that this technique is feasible in well-selected patients when performed by experienced laparoscopic hepatobiliary surgeons. All previous reports concluded that correct patient selection and laparoscopy experience are the most important part of a successful outcome [10-15].

Acknowledgments We thank Ali Kumbasar for the photographs. www.scribendi.com made English language editing of the manuscript. This retrospective study is approved by Ondokuz mayis University Human Research Ethics Committee (OMU KAEK 2015/299).

\section{Compliance with Ethical Standards}

Conflict of Interest There is no conflict of interest to declare.

\section{References}

1. Nguyen KT, Gamblin TC, Geller DA (2009) World review of laparoscopic liver resection-2,804 patients. Ann Surg 250: 831-841

2. Castaing D, Vibert E, Ricca L, Azoulay D, Adam R, Gayet B (2009) Oncologic results of laparoscopic versus open hepatectomy for colorectal liver metastases in two specialized centers. Ann Surg 250:849-855

3. Tzanis D, Shivathirthan N, Laurent A, Abu Hilal M, Soubrane O, Kazaryan AM et al (2013) European experience of laparoscopic major hepatectomy. J Hepatobiliary Pancreat Sci 20:120-124

4. Belli G, Fantini C, D'Agostino A, Cioffi L, Langella S, Russolillo $\mathrm{N}$ et al (2007) Laparoscopic versus open liver resection for hepatocellular carcinoma in patients with histologically proven cirrhosis: short- and middle-term results. Surg Endosc 21(11):2004-2011

5. Cherqui D, Laurent A, Tayar C, Chang S, Nhieu JT, Loriau J (2006) Laparoscopic liver resection for peripheral hepatocellular carcinoma in patients with chronic liver disease: midterm results and perspectives. Ann Surg 243:499-506

6. Rao PP, Rao PP, Bhagwat S (2011) Single-incision laparoscopic surgery - current status and controversies. J Minim Access Surg 7(1):6-16

7. Rivas H, Varela E, Scott D (2010) Single-incision laparoscopic cholecystectomy: initial evaluation of a large series of patients. Surg Endosc 24:1403-1412 
8. Katsuno G, Fukunaga M, Nagakari K, Yoshikawa S, Ouchi M, Hirasaki Y (2011) Single-incision laparoscopic colectomy for colon cancer: early experience with 31 cases. Dis Colon Rectum 54(6): 705-710

9. Mittermair R, Pratschke J, Sucher R (2013) Single-incision laparoscopic sleeve gastrectomy. Am Surg 79(4):393-397

10. Shetty GS, You YK, Choi HJ, Na GH, Hong TH, Kim DG (2012) Extending the limitations of liver surgery: outcomes of initial human experience in a high-volume center performing single-port laparoscopic liver resection for hepatocellular carcinoma. Surg Endosc 26:1602-1608

11. Tayar C, Subar D, Salloum C, Malek A, Laurent A, Azoulay D (2014) Single incision laparoscopic hepatectomy: advances in laparoscopic liver surgery. J Minim Access Surg 10(1):14-17

12. Chang SK, Mayasari M, Ganpathi IS, Wen VL, Madhavan K (2011) Single port laparoscopic liver resection for hepatocellular carcinoma: a preliminary report. Int J Hepatol 2011:579203

13. Gkegkes ID, Iavazzo C (2014) Single incision laparoscopic hepatectomy: a systematic review. J Minim Access Surg 10(3):107-112
14. Aikawa M, Miyazawa M, Okamoto K, Toshimitsu Y, Okada K, Ueno Y et al (2012) Single-port laparoscopic hepatectomy: technique, safety, and feasibility in a clinical case series. Surg Endosc 26:1696-1701

15. Hu M, Zhao G, Wang F, Xu D, Liu R (2014) Single-port and multiport laparoscopic left lateral liver sectionectomy for treating benign liver diseases: a prospective, randomized, controlled study. World J Surg 38(10):2668-2673

16. Karabicak I, Yuruker S, Seren DT, Kesicioglu T, Cinar H, Ozen N (2013) Single incision laparoscopic surgery for hepatic hydatid disease. Report of a case. Ann Ital Chir 84(4):451-453

17. Choi HJ, You YK, Na GH, Hong TH, Shetty GS, Kim DG (2012) Single-port laparoscopy-assisted donor right hepatectomy in living donor liver transplantation: sensible approach or unnecessary hindrance? Transplant Proc 44(2):347-352

18. Belli G, Fantini C, D'Agostino A, Cioffi L, Russo G, Belli A et al (2011) Laparoendoscopic single site liver resection for recurrent hepatocellular carcinoma in cirrhosis: first technical note. Surg Laparosc Endosc Percutan Tech 21(4):e166-e168 